\title{
[gw22-e0867] THE RISK FACTORS OF CONTRAST-INDUCED NEPHROPATHY IN PATIENTS UNDERGOING PRIMARY PERCUTANEOUS CORONARY INTERVENTION
}

QiaoPing, Wangsheng, Wangwei, Chengguanliang, Libin, Wuming Department of Cardiology, Hainan Provincial Hospital, China

10.1136/heartjnl-2011-300867.494

Objective To analyse the effect of contrast medium on renal function in patients undergoing primary percutaneous coronary intervention (PCI), and to assess the clinical predictors of contrast-induced nephropathy (CIN).

Methods In 167 patients who undergoing primary percutaneous coronary intervention, serum creatinine concentration (Scr) was measured at baseline and on 48th $\mathrm{h}, 72 \mathrm{nd} \mathrm{h}$ after PCI.

Results CIN occurred in 32 (19.16\%) patients. The baseline glomerular filtration rate (GFR) and left ventricular ejection fraction (LVEF) was lower in CIN group than that in non-CIN group ( $p<0.05)$, the contrast volume was higher in CIN group than that in non-CIN group $(\mathrm{p}<0.001)$. Multivariate analysis showed that GFR, Scr, LVEF, Age, contrast volume, multivariate arterial disease were correlated with CIN.

Conclusions The most significant risk factors appear to be GFR. LVEF, age, contrast volume, multivariate arterial disease are all independent predictor of CIN development after PCI. 